\title{
SÓ ERRA QUEM FAZ, E QUEM FAZ ERRA! UM MANIFESTO PELO CÁLCULO DA PROPAGAÇÃO DE INCERTEZAS
}

\author{
Luís Moreira Gonçalves ${ }^{\mathrm{a}, *,(1)}$ \\ a Departamento de Química Fundamental, Instituto de Química, Universidade de São Paulo, 05508-000 São Paulo - SP, Brasil
}

Recebido em 31/07/2019; aceito em 07/10/2019; publicado na web em 14/01/2020

\begin{abstract}
ONLY THOSE WHO EXPERIMENT MAKE ERRORS, AND WHO EXPERIMENTS, ERRS! A MANIFESTO FOR THE CALCULATION OF THE PROPAGATION OF UNCERTAINTIES. Making experimental measurements, whether we are at peace in the face of such a reality or not, invariably involves experimental uncertainty. Unfortunately, presenting uncertainty alongside the results is not as widespread as it should be. The author attempts here to simplify, improve understanding, and appeal to a healthy living with uncertainty. One of the main tools scientists should know that there is available is the uncertainty propagation equation, which can be applied to all equations involving experimental results.
\end{abstract}

Keywords: central limit theorem; experimental errors; normal distribution; propagation of uncertainty.

\section{INTRODUÇÃO}

Há uma frase comum, quando o aluno motivado, mas ainda desajeitado, parte alguma coisa no laboratório:

- "Só erra quem faz!"

E aqui, o autor deste artigo, gostaria de acrescentar:

- "E quem faz erra!".

As medidas experimentais têm, invariavelmente, erros. No limite, esta afirmação pode ser justificada com o princípio de incerteza de Heisenberg, que refere que quanto menor for a incerteza na medida da posição de uma partícula, maior será a incerteza na medição seu momento linear e vice-versa. ${ }^{1}$ Sendo assim, quando se apresenta o resultado de uma medição, e, obviamente, quando se apresentam as conclusões deduzidas desses resultados, deve vir associado uma incerteza. Esse conceito pode ser subliminar, mas pode também, e até é preferível, ser explícito e quantificado:

$$
x \pm \Delta x
$$

onde $x$ é o resultado experimental e $\Delta x$ é a incerteza do resultado experimental.

O autor deste artigo é professor, bem como um leitor e revisor de artigos científicos, além de um leitor habitual de reportagens e livros com conteúdo científico. Em todas estas atividades é surpreendente o número de vezes que não se apresenta qualquer tipo de incerteza. E é uma pena. A manifestação de incertezas não é um reflexo de ignorância ou falta de profissionalismo. Pelo contrário, demonstra sapiência e demonstra sabedoria das limitações do conhecimento. E essa clarividência Socrática é fundamental para um bom pesquisador.

No geral, os cientistas têm uma boa noção de incerteza. Então, porque é tão difícil, quando expressam um resultado, associarem uma incerteza? É difícil identificar a origem do problema, talvez seja devido a uma antipatia natural para com o erro, talvez os livros (incluindo muitos dos livros de referência em química analítica) não deduzam a fórmula de propagação de incerteza ou adequadamente demonstrem a sua impressionante aplicabilidade, talvez seja pelo receio dos professores em expor os alunos a tanta matemática nas suas aulas. $\mathrm{O}$ autor não sabe, mas mesmo sem saber a causa, lamenta, e espera, contudo, que este manuscrito contribua um pouco para mudar a situação.

*e-mail: lmgoncalves@iq.usp.br

\section{Teorema central do limite e 'normalidade'}

O teorema central do limite (TCL) talvez seja um bom ponto de partida. É um dos conceitos mais importantes em estatística (e até mesmo em toda a matemática), e é aqui importante porque a sua premissa nos 'autoriza' calcular a incerteza. O TCL refere que retirando algumas amostras de uma população (em particular um número elevado de amostras), a função de probabilidade (fdp) das médias das amostras adquire o formato de uma curva de distribuição normal. Uma função normal tem por base esta equação:

$$
f(x)=\frac{1}{\sigma \sqrt{2 \pi}} e^{-\frac{1}{2}\left(\frac{x-\mu}{\sigma}\right)^{2}}
$$

onde $\mu$ é a média, e $\sigma$ é o desvio-padrão, calculado por:

$$
\sigma=\sqrt{\frac{1}{N} \sum_{i=1}^{N}\left(x_{i}-\mu\right)^{2}}
$$

Embora a história da distribuição normal seja fora do âmbito deste artigo é muito interessante, e o leitor mais interessado é aconselhado a ler sobre o tópico. ${ }^{2}$

Explicando o TCL de forma mais elegante, a média amostral $(\bar{X})$ é a soma de $n$ dados $\left(S_{n}\right)$ a dividir por $n$ :

$$
\bar{X}=\frac{X_{1}+X_{2}+\ldots+X_{n}}{n}=\frac{1}{n} \sum_{i=1}^{n} X_{i}=\frac{S_{n}}{n}
$$

O TCL estabelece que, com um valor de $n$ elevado, a distribuição de $\bar{X}$ converge para uma distribuição normal padrão:

$$
\bar{X} \sim N\left(\mu, \frac{\sigma^{2}}{n}\right)
$$

Isto porque na notação matemática mais comum: $Y \sim N(a, b)$, significa que a variável $Y$ segue uma distribuição $(\sim)$ normal $(N)$ de média $a$ e variância $b$ ).

Para evitar a degeneração que ocorre à variância $\left(\frac{\sigma^{2}}{n}\right)$ quando $n$ tende para o infinito (pois variância passa a ser zero). Representamos:

$$
Z=\frac{\bar{X}-\mu}{\sigma / \sqrt{n}}=\frac{S_{n}-n \mu}{\sqrt{n} \sigma}=\frac{\sum_{i=1}^{n} x_{i}-n \mu}{\sqrt{n} \sigma}
$$

Finalmente, a TCL estabelece que a distribuição de $Z_{n}$ converge para uma distribuição normal padrão quando o $n$ tende para o 
infinito, com valor esperado de média de $0(E[Z n]=0)$ e variância de $1\left(\operatorname{Var}\left(Z_{n}\right)=1\right)$.

$$
\underset{n \rightarrow \infty}{Z} \sim N(0,1)
$$

O quão grande o número de amostras precisa ser depende do quão normal é a distribuição original. Se a distribuição original for próxima de uma curva 'formato de sino' basta um $n$ de 10; para populações mais anormais alguns autores referem um $n$ de 50; Outros simplificam e referem $n$ de 30 independentemente da população. ${ }^{3}$

Como foi dito previamente, a fdp das médias das amostras adquire o formato de uma curva de distribuição normal, e se levarmos em conta a equação 2 acima descrita de uma função normal padrão, teremos que:

$$
f d p=\frac{1}{\sqrt{2 \pi}} e^{-\frac{z^{2}}{2}}
$$

Um detalhe importante, a área de toda a curva fdp é sempre 1, o fator $\frac{1}{\sqrt{2 \pi}}$ garante isso, com pontos de inflexão - 1 e 1 , ou seja:

$$
\int_{-\infty}^{+\infty} \frac{1}{\sqrt{2 \pi}} e^{-\frac{z^{2}}{2}} d z=1
$$

O TCL é particularmente impressionante pois é aplicável mesmo a populações que não estão normalmente distribuídas (Figura 1). ${ }^{3}$

\section{A propagação de incerteza per se}

Após este pequeno introito sobre o TCL, podemos ir agora diretamente para a propagação de incerteza. Seja, então, $Y$ uma variável calculada como função de várias variáveis aleatórias $\left(X_{1}, X_{2}, \ldots, X_{n}\right)$.

$$
Y=f\left(X_{1}, X_{2}, \ldots, X_{n}\right)
$$

E considere-se que estas variáveis possuem uma distribuição normal de média $\mu_{i}$ e desvio padrão $\sigma_{i}$ conhecidos. Se os desvios padrão $\sigma_{i}$ forem suficientemente pequenos, a função $f$ pode ser desenvolvida numa série de Taylor em torno dos valores médios $\left\{\mu_{i}\right\}$ e truncada nos termos lineares. ${ }^{3}$

$$
Y=f\left(X_{1}, X_{2}, \ldots, X_{n}\right)_{\left\{\mu_{i}\right\}}+\sum_{i=1}^{n}\left(X_{i}-\mu_{i}\right)\left(\frac{\partial f}{\partial X_{i}}\right)_{\left\{\mu_{i}\right\}}
$$

Desta forma os parâmetros da variável aleatória $Y$ podem ser facilmente determinados:

a) média:

$$
E[Y]=E\left[f\left(X_{1}, X_{2}, \ldots, X_{n}\right)_{\left\{\mu_{i}\right\}}+\sum_{i=1}^{n}\left(X_{i}-\mu_{i}\right)\left(\frac{\partial f}{\partial X_{i}}\right)_{\left\{\mu_{i}\right\}}\right]
$$

E como:

$$
E\left[X_{i}-\mu_{i}\right]=E\left[X_{i}\right]-\mu_{i}=0
$$

Temos que:

$$
E[Y]=f\left(X_{1}, X_{2}, \ldots, X_{n}\right)_{\left\{\mu_{i}\right\}}=\mu_{Y}
$$

b) desvio-padrão

(lembrar que: $\left.(a+b)^{2}=a^{2}+2 a b+b^{2}\right)$ :

$\sigma_{Y}=\sqrt{\operatorname{Var}(Y)}=\sqrt{E\left[\left(Y-\mu_{Y}\right)^{2}\right]}=\sqrt{E\left[Y^{2}-2 Y \mu_{Y}+\mu_{Y}^{2}\right]}=$

$\sqrt{E\left[\left(\mu_{Y}+\sum_{i=1}^{n}\left(X_{i}-\mu_{i}\right)\left(\frac{\partial f}{\partial X_{i}}\right)_{\left\{u_{\}}\right\}}\right)^{2}-2\left(\mu_{Y}+\sum_{i=1}^{n}\left(X_{i}-\mu_{i}\right)\left(\frac{\partial f}{\partial X_{i}}\right)_{\left\{\mu_{1}\right\}}\right) \mu_{Y}+\mu_{Y}^{2}\right]}=$

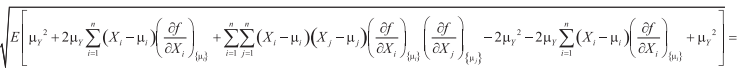

$\sqrt{E\left[\sum_{i=1}^{n} \sum_{j=1}^{n}\left(X_{i}-\mu_{i}\right)\left(X_{j}-\mu_{j}\right)\left(\frac{\partial f}{\partial X_{i}}\right)_{\{\mu,\}}\left(\frac{\partial f}{\partial X_{j}}\right)_{\{\mu,\}}\right]}=$

$\sqrt{\sum_{i=1}^{n} \sum_{j=1}^{n}\left(\frac{\partial f}{\partial X_{i}}\right)_{\{\mu,\}}\left(\frac{\partial f}{\partial X_{j}}\right)_{\{\mu, j\}} E\left[\left(X_{i}-\mu_{i}\right)\left(X_{j}-\mu_{j}\right)\right]}=\sqrt{\sum_{i=1}^{n} \sum_{j=1}^{n}\left(\frac{\partial f}{\partial X_{i}}\right)_{\{\mu,\}}\left(\frac{\partial f}{\partial X_{j}}\right)_{\left\{\mu_{j}\right\}} V_{i j}}$

ou seja:

$$
\sigma_{Y}=\sqrt{\sum_{i=1}^{n} \sum_{j=1}^{n}\left(\frac{\partial f}{\partial X_{i}}\right)_{\left\{\mu_{i}\right\}}\left(\frac{\partial f}{\partial X_{j}}\right)_{\left\{\mu_{j}\right\}} V_{i j}}
$$

onde:

$$
V_{i j}=\left[\begin{array}{ccc}
X_{1}^{i} X_{1}^{j} & \cdots & X_{1}^{i} X_{n}^{j} \\
\vdots & \ddots & \vdots \\
X_{n}^{i} X_{1}^{j} & \cdots & X_{n}^{i} X_{n}^{j}
\end{array}\right]
$$

Onde $V$ é a matriz de variância-covariância de uma função $Y$. Na prática, esta matriz infere como as diferentes variáveis interferem entre si, ou seja, quantifica o grau de não-independência.

A equação 16 também aparece elegantemente representada na literatura, como: ${ }^{4}$

$$
\sigma_{Y}=\sqrt{g^{T} V g}
$$

onde $g$ é um vetor e $g^{T}$ é o vetor transposto.

Se assumirmos que as variáveis são independentes, ou seja, sem covariâncias e $V_{i j}$ for igual a 1 .

$$
\sigma_{Y}=\sqrt{\sum_{i=1}^{n}\left(\frac{d y}{d x_{i}}\right)^{2}\left(\Delta x_{i}\right)^{2}}
$$

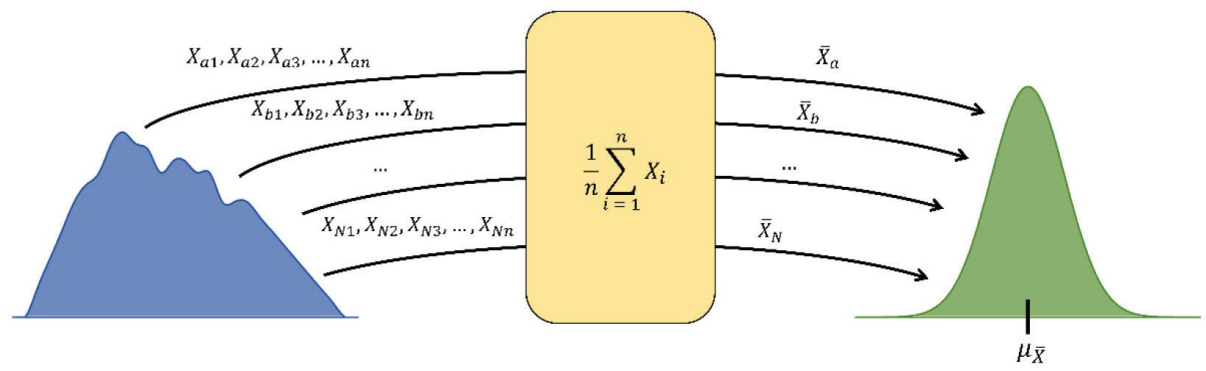

Figura 1. Representação esquemática do TCL. É calculada a média de cada uma das diferentes amostras retiradas da população não normal (no lado esquerdo). A distribuição dessas mesmas médias é, interessantemente, normal (no lado direito) 
Para simplificarmos, vamos considerar que $\sigma_{Y}=\Delta y$ :

$$
\Delta y=\sqrt{\sum_{i}\left(\frac{\delta y}{\delta x_{i}}\right)^{2}\left(\Delta x_{i}\right)^{2}}
$$

E esta é talvez a equação mais relevante do tópico deste manuscrito, a equação de propagação de erros. Se o leitor deste artigo não a decorar, por favor coloque-a em grande na parede, colada com fita-adesiva.

Para estudantes que ainda não tenham feito Cálculo, é o mesmo que:

$\Delta y=\sqrt{\left(\frac{d y}{d x_{1}}\right)^{2}\left(\Delta x_{1}\right)^{2}+\left(\frac{d y}{d x_{2}}\right)^{2}\left(\Delta x_{2}\right)^{2}+\left(\frac{d y}{d x_{3}}\right)^{2}\left(\Delta x_{3}\right)^{2}+\ldots+\left(\frac{d y}{d x_{n}}\right)^{2}\left(\Delta x_{n}\right)^{2}}$

Estão a ser usadas derivadas parciais, ou seja, $\frac{d y}{d x_{1}}$ é a derivada da função $y$ em relação à variável $x_{1}$ assumindo todas as outras variáveis como constantes $\left(x_{1}, x_{2}, x_{3}, \ldots, x_{n}\right)$, e $\frac{d y}{d x_{2}}$ é a derivada da função $y$ em relação à variável $x_{2}$, assumindo todås as outras variáveis como constantes $\left(x_{1}, x_{2}, x_{3}, \ldots, x_{n}\right)$; e assim sucessivamente.

Vejamos o que se passa quando aplicamos a equação de propagação de incertezas a alguns dos casos principais:

Na multiplicação com uma constante:

$$
y=k a
$$

(sendo $k$ uma constante), teremos que (lembrar que: $\frac{d}{d x} k x=k$ ).

$\Delta y=\sqrt{\left(\frac{\partial}{\partial a} k a\right)^{2}(\Delta a)^{2}}=\sqrt{(k)^{2}(\Delta a)^{2}}=k \Delta a$

Na adição:

$$
y=a+b
$$

teremos que (lembrar que: $\frac{d}{d x} x^{n}=n x^{n-1}$; ergo: $\frac{d}{d x} x=1$ ).

$$
\begin{gathered}
\Delta y=\sqrt{\left(\frac{\partial}{\partial a}(a+b)\right)^{2}(\Delta a)^{2}+\left(\frac{\partial}{\partial b}(a+b)\right)^{2}(\Delta b)^{2}}= \\
\sqrt{1^{2}(\Delta a)^{2}+1^{2}(\Delta b)^{2}}=\sqrt{(\Delta a)^{2}+(\Delta b)^{2}}
\end{gathered}
$$

Na subtração:

$$
y=a-b
$$

teremos que:

$$
\begin{gathered}
\Delta y=\sqrt{\left(\frac{\partial}{\partial a}(a-b)\right)^{2}(\Delta a)^{2}+\left(\frac{\partial}{\partial b}(a-b)\right)^{2}(\Delta b)^{2}}= \\
\sqrt{1^{2}(\Delta a)^{2}+(-1)^{2}(\Delta b)^{2}}=\sqrt{(\Delta a)^{2}+(\Delta b)^{2}}
\end{gathered}
$$

Na multiplicação:

$$
y=a b
$$

teremos que:

$$
\begin{gathered}
\Delta y=\sqrt{\left(\frac{\partial}{\partial a}(a b)\right)^{2}(\Delta a)^{2}+\left(\frac{\partial}{\partial b}(a b)\right)^{2}(\Delta b)^{2}}=\sqrt{(b)^{2}(\Delta a)^{2}+(a)^{2}(\Delta b)^{2}}= \\
\sqrt{\left(\frac{y}{a}\right)^{2}(\Delta a)^{2}+\left(\frac{y}{b}\right)^{2}(\Delta b)^{2}}=y \sqrt{\left(\frac{\Delta a}{a}\right)^{2}+\left(\frac{\Delta b}{b}\right)^{2}}
\end{gathered}
$$

Na divisão:

$$
y=\frac{a}{b}
$$

teremos que:

$$
\begin{gathered}
\Delta y=\sqrt{\left(\frac{\partial}{\partial a}\left(\frac{a}{b}\right)\right)^{2}(\Delta a)^{2}+\left(\frac{\partial}{\partial b}\left(\frac{a}{b}\right)\right)^{2}(\Delta b)^{2}}=\sqrt{\left(\frac{1}{b}\right)^{2}(\Delta a)^{2}+\left(-\frac{a}{b^{2}}\right)^{2}(\Delta b)^{2}}= \\
\sqrt{\left(\frac{y}{a}\right)^{2}(\Delta a)^{2}+\left(-\frac{a}{b^{2}}\right)^{2}(\Delta b)^{2}}=\sqrt{\frac{y^{2}}{a^{2}}(\Delta a)^{2}+\frac{a^{2}}{b^{2} b^{2}}(\Delta b)^{2}}= \\
\sqrt{\frac{y^{2}}{a^{2}}(\Delta a)^{2}+\frac{y^{2}}{b^{2}}(\Delta b)^{2}}=y \sqrt{\left(\frac{\Delta a}{a}\right)^{2}+\left(\frac{\Delta b}{b}\right)^{2}}
\end{gathered}
$$

Com potências:

\begin{tabular}{|c|c|}
\hline & $\left.\frac{\delta y}{\delta x_{i}}\right)^{2}\left(\Delta x_{i}\right)^{2}$ \\
\hline$y=k a$ & $\Delta y=k \Delta a$ \\
\hline$y=a+b$ & $\Delta y=\sqrt{(\Delta a)^{2}+(\Delta b)^{2}}$ \\
\hline$y=a-b$ & $\Delta y=\sqrt{(\Delta a)^{2}+(\Delta b)^{2}}$ \\
\hline$y=a b$ & $\Delta y=y \sqrt{\left(\frac{\Delta a}{a}\right)^{2}+\left(\frac{\Delta b}{b}\right)^{2}}$ \\
\hline$y=\frac{a}{b}$ & $\Delta y=y \sqrt{\left(\frac{\Delta a}{a}\right)^{2}+\left(\frac{\Delta b}{b}\right)^{2}}$ \\
\hline$y=k^{a}$ & $\Delta y=y \ln (a) \Delta a$ \\
\hline$y=\log _{k} a$ & $\Delta y=\frac{\Delta a}{\ln (k) a}$ \\
\hline
\end{tabular}

$$
y=k^{a}
$$

(sendo $k$ uma constante), teremos que (lembrar que: $\frac{d}{d x} k^{x}=k^{x} \ln (k)$ )

$$
\begin{gathered}
\Delta y=\sqrt{\left(\frac{\partial}{\partial a} k^{a}\right)^{2}(\Delta a)^{2}}=\sqrt{\left(k^{a} \ln (a)\right)^{2}(\Delta a)^{2}}= \\
\sqrt{y^{2}(\ln (a))^{2}(\Delta a)^{2}}=y \ln (a) \Delta a
\end{gathered}
$$

Com logaritmos:

$$
y=\log _{k} a
$$

(sendo $k$ uma constante), teremos que (lembrar que:

$\left.\frac{d}{d x} \log _{k} x=\frac{1}{x} \log _{k}(e)=\frac{1}{x \ln (k)}\right)$.

$\Delta y=\sqrt{\left(\frac{\partial}{\partial a} \log _{k} a\right)^{2}(\Delta a)^{2}}=\sqrt{\left(\frac{1}{a \ln (k)}\right)^{2}(\Delta a)^{2}}=\frac{\Delta a}{\ln (k) a}$

Estas equações estão resumidas na Tabela 1.

Tabela 1. Quadro resumo das fórmulas a aplicar no cálculo das incertezas

\section{ALGUMAS NOTAS SOBRE A APLICAÇÃO DA PROPAGAÇÃO DE INCERTEZA NA QUÍMICA}

Podemos começar por ver, na Tabela 2, algumas aplicações diretas das fórmulas da Tabela 1 em alguns exemplos práticos na vida de um químico.

Mas é importante perceber que a equação de propagação de erros não se aplica apenas em casos de aplicação mais trivial como os descritos na tabela. Pode ser sempre aplicada. Seguem alguns exemplos 
Tabela 2. Alguns exemplos práticos de aplicação do cálculo da incerteza

$$
\Delta y=\sqrt{\sum_{i}\left(\frac{\delta f}{\delta x_{i}}\right)^{2}\left(\Delta x_{i}\right)^{2}}
$$

\begin{tabular}{cc}
$\begin{array}{c}A b s=\varepsilon b[X] \text { (assumindo } \varepsilon \mathrm{e} \mathrm{b} \\
\text { como constantes) } \\
n_{\text {total }}=n_{1}+n_{2}\end{array}$ & $\Delta A b s=\varepsilon b \Delta[X]$ \\
$m_{x}=m_{\text {final }}-m_{\text {inicial }}$ & $\Delta n_{\text {total }}=\sqrt{\left(\Delta n_{1}\right)^{2}+\left(\Delta n_{2}\right)^{2}}$ \\
$V=R I$ & $\Delta m_{x}=\sqrt{\left(\Delta m_{\text {final }}\right)^{2}+\left(\Delta m_{\text {inicial }}\right)^{2}}$ \\
{$[A]=\frac{n_{A}}{V}$} & $\Delta V=V \sqrt{\left(\frac{\Delta R}{R}\right)^{2}+\left(\frac{\Delta I}{I}\right)^{2}}$ \\
$T=10^{-\mathrm{Abs}}$ & $\Delta[H A]=[A] \sqrt{\left(\frac{\Delta n_{A}}{n_{A}}\right)^{2}+\left(\frac{\Delta V}{V}\right)^{2}}$ \\
$p H=\log \left[H^{+}\right]$ & $\Delta T \cong 2,303 T \Delta A b s$ \\
\hline
\end{tabular}

(o autor desafia o leitor mais interessado a fazer a demonstração por si mesmo antes de verificar a mesma em informação suplementar):

$$
\begin{gathered}
V=l^{3} \rightarrow \Delta V=3 l^{2} \Delta l \\
s=\sqrt{K_{p s}} \rightarrow \Delta s=\frac{\Delta K_{p s}}{2 \sqrt{K_{p s}}} \\
P=\frac{n R T}{V} \rightarrow \Delta y=P \sqrt{\left(\frac{\Delta n}{n}\right)^{2}+\left(\frac{\Delta T}{T}\right)^{2}+\left(\frac{\Delta V}{V}\right)^{2}} \text { se } \Delta R=0 \\
V=\frac{4}{3} \pi r^{3} \rightarrow \Delta V=4 \pi r^{2} \Delta r
\end{gathered}
$$

Existem já, vários sites para calcular a incerteza, ${ }^{5-7} \mathrm{e}$, inclusive, aplicativos para dispositivos móveis. Um bom exemplo, é o aplicativo para Android 'Error Propagation Calculator' criado por Julian Wien, quando estudava na Universidade de Würzburg na Alemanha. ${ }^{8}$

Imagine que temos de preparar um litro de uma solução tampão de fosfato, $\mathrm{pH} 7.4,0,1 \mathrm{~mol} \mathrm{~L}^{-1}$. Fazemos as contas e chegamos à conclusão que temos de pesar 20,210 g de hidrogenofosfato de sódio heptahidratado $\left(m_{1}\right)$, massa molar de 268,07 $\mathrm{g} \mathrm{mol}^{-1}\left(M M_{1}\right)$, e 3,395 g dihidrogenofosfato de sódio monohidratado $\left(m_{2}\right)$, massa molar de $137,99 \mathrm{~g} \mathrm{~mol}^{-1}\left(M M_{2}\right)$. A incerteza da concentração $(\Delta C)$ é o seguinte (a demonstração encontra-se na informação suplementar):

$\Delta C=C \sqrt{\left.\frac{\sqrt{\left(\frac{\Delta m_{1}}{M M_{1}}\right)^{2}+\left(\frac{\Delta m_{2}}{M M_{2}}\right)^{2}}}{\frac{m_{1}}{M M_{1}}+\frac{m_{2}}{M M_{2}}}\right)^{2}+\left(\frac{\Delta V}{V}\right)^{2}}$

Para esta fórmula não foi levado em conta a possível adição de ácido ou base para acertar o $\mathrm{pH}$.

Se optarmos por fazer a solução usando uma balança analítica $( \pm 0,1 \mathrm{mg})$ e um balão volumétrico classe $\mathrm{A}( \pm 0,3 \mathrm{~mL})$ teremos de concentração com a respetiva incerteza: $(0,09999 \pm 0,00003) \mathrm{mol} \mathrm{L}^{-1}$. Se pelo contrário, for utilizada uma balança semi-analítica $( \pm 1 \mathrm{mg})$ e um béquer comum $( \pm 25 \mathrm{~mL})$, teremos no final: $(0,100 \pm 0,002)$ mol L ${ }^{-1}$. Normalmente, o recurso a uma solução tampão é apenas para tamponar o meio, não é muito importante ter uma concentração muito precisa. Como tal, é justificado que se use um béquer e uma balança mais acessível. Deste modo, não só a preparação da solução é mais rápida e simples mas também não utilizamos material mais frágil.

Pensemos agora num exemplo antagónico. A titulação de $25 \mathrm{~mL}$ $\left(V_{\text {titulado }}\right)$ de um ácido forte monoprótico com hidróxido de sódio de concentração $\left(C_{\text {titulante }}\right)$ conhecida, $(0,1223 \pm 0,0002) \mathrm{mol} \mathrm{L}^{-1}$.

$$
\Delta C_{\text {titulado }}=C_{\text {titulado }} \sqrt{\left(\frac{\Delta C_{\text {titulante }}}{C_{\text {titulante }}}\right)^{2}+\left(\frac{\sqrt{\left(\Delta V_{f}\right)^{2}+\left(\Delta V_{i}\right)^{2}}}{V_{f}-V_{i}}\right)^{2}+\left(\frac{\Delta V_{\text {titulado }}}{V_{\text {titulado }}}\right)^{2}}
$$

Se a titulação $\left(V_{f}\right.$ de $42 \mathrm{~mL}$ e $V_{i}$ de $\left.9 \mathrm{~mL}\right)$ for feita de forma normal com uma bureta $\left(\Delta V_{f}=\Delta V_{i}=0,02 \mathrm{~mL}\right)$ e o titulado for medido com uma pipeta volumétrica $\left(\Delta V_{\text {titulado }}\right.$ de $\left.0,02 \mathrm{~mL}\right)$, teremos que: $(0,1614$ $\pm 0,0003) \mathrm{mol} \mathrm{L}^{-1}$. Se, porventura a "titulação" for feita com menos cuidado utilizando uma proveta $\left(\Delta V_{f}=\Delta V_{i}=1 \mathrm{~mL}\right)$ e o titulado for medido com um béquer $\left(\Delta V_{\text {titulado }}\right.$ de $\left.3 \mathrm{~mL}\right)$, teremos que: $(0,16 \pm$ $0,02) \mathrm{mol} \mathrm{L}^{-1}$, ou seja mais de $10 \%$ de incerteza. Neste caso, querendo conhecer a concentração do ácido com precisão faz mais sentido usar a bureta e uma pipeta volumétrica.

Estes dois exemplos demonstram que, sabendo como calcular a incerteza, podemos pensar como melhor realizar os experimentos, decidindo investir, ou não, em recursos para aumentar a precisão da nossa análise. É importante entender que é relevante não só calcular a incerteza como compreender a incerteza.

\section{MATERIAL SUPLEMENTAR}

As demonstrações das equações da Tabela 2 (Parte 1), bem como das equações 40 e 41 (Parte 2) estão disponíveis em http://quimicanova.sbq.org.br, com acesso livre.

\section{CONCLUSÃO}

Só para ficar inequivocamente claro: é impossível realizar uma análise química que não tenha uma incerteza associada. Quem faz erra! Mas os químicos, e outros cientistas, têm uma arma poderosíssima à sua disposição, a equação de propagação de incerteza. $\mathrm{O}$ autor apela que a compreendam e a usem com discernimento na produção de ciência.

\section{AGRADECIMENTOS}

O autor agradece o apoio da Coordenação de Aperfeiçoamento de Pessoal de Nível Superior - Brasil (CAPES) e Fundação de Amparo à Pesquisa do Estado de São Paulo (FAPESP - 2018/14425-7). O autor agradece também aos alunos do grupo de pesquisa que se disponibilizaram para rever o artigo.

\section{REFERÊNCIAS}

1. Heisenberg, W.; Zeitschrift fur Phys. 1927, 43, 172.

2. Stahl, S.; Math. Mag. 2006, 79, 96.

3. Cordeiro, N.; Magalhães, A.; Introdução à Estatística - Uma perspectiva química; 1st ed.; Lidel: Porto, 2004.

4. Tellinghuisen, J.; J. Phys. Chem. A 2001, 105, 3917.

5. Error propagation calculator https://www.eoas.ubc.ca/courses/eosc252/ error-propagation-calculator-fj.htm (accessed Apr 18, 2019).

6. Propagation of error (uncertainty) https://www.wolframalpha.com/ widgets/view.jsp?id=8ac60957610e1ee4894b2cd58e753 (accessed Apr 18, 2019).

7. Uncertainty Calculator http://web.mst.edu/ gbert/JAVA/uncertainty.html (accessed Apr 18, 2019).

8. Julianibus http://julianibus.de/ (accessed Apr 18, 2019). 